\title{
Kernos
}

Revue internationale et pluridisciplinaire de religion grecque antique

$21 \mid 2008$

Varia

\section{Cornelia ISLER-KERÉNYI, Dionysos in Archaic Greece. An Understanding through Images}

\section{Anne-Françoise Jaccottet}

\section{OpenEdition \\ Journals}

Édition électronique

URL : https://journals.openedition.org/kernos/1687

DOI : 10.4000/kernos. 1687

ISSN : 2034-7871

\section{Éditeur}

Centre international d'étude de la religion grecque antique

\section{Édition imprimée}

Date de publication : 1 janvier 2008

Pagination : $327-330$

ISSN : 0776-3824

Référence électronique

Anne-Françoise Jaccottet, " Cornelia ISLER-KERÉNYI, Dionysos in Archaic Greece. An Understanding through Images », Kernos [En ligne], 21 | 2008, mis en ligne le 15 septembre 2011, consulté le 24 août 2022. URL : http://journals.openedition.org/kernos/1687 ; DOI : https://doi.org/10.4000/kernos.1687 
En conclusion, cette synthèse rassemble commodément les principales questions posées sur les dieux grecs et les réponses qui ont été données au fil du temps, sans toutefois en dresser un bilan historiographique. Il n'intéressera donc que de manière limitée les spécialistes du domaine. D'autre part, s'il s'adresse à un public cultivé, cet ouvrage n'est pas pour autant accessible à tous et nécessite, au contraire, une discrète connaissance préliminaire des sujets traités, l'A. ayant essayé de concilier concision et complexité au lieu de simplifier l'argumentation à des fins pédagogiques. Quoi qu'il en soit, même si l'expérience n'est pas complètement réussie, on peut saluer la perspective adoptée par l'A. qui, ayant choisi comme point de départ la représentation du divin, offre à ses lecteurs une image du polythéisme qui, à défaut d'être exhaustive ou novatrice, a néanmoins le mérite de restituer aux divinités la place centrale qu'elles occupaient dans la vie religieuse des Grecs.

Gabriella PIRONTI

(Université de Liège - Centre Louis Gernet-Paris)

\section{Cornelia IsLER-KerÉNYI, Dionysos in Archaic Greece. An Understanding through} Images, translated by Wilfred G.E. Watson, Leiden, Brill, 2007. 1 vol. $16 \times$ 24, $5 \mathrm{~cm}, \mathrm{XX}+291$ p. (Religions in the Graeco-Roman World, 160). ISBN : 90-0414445-5.

Comprendre par le biais des images le rôle religieux et social du Dionysos archaïque, tel est le défi auquel s'est attaquée l'A. en 2001 dans une étude parue en italien et dont l'ouvrage discuté ici, paru en 2007, est une traduction anglaise, augmentée et partiellement mise à jour. C'est en se positionnant clairement en archéologue plutôt qu'en historienne des religions que C.I.-K. aborde cette délicate question, choisissant une approche rigoureusement chronologique (presque stratigraphique) et prenant grand soin de contextualiser chaque image par rapport à son support (type de vase) et par là-même à son environnement d'utilisation. L'étude se démarque encore par la volonté d'ouvrir le champ d'investigation à d'autres zones de production céramique qu'Athènes, comme au proto-corinthien et au corinthien moyen, aux productions archaïques béotiennes, laconiennes et ioniennes. L'athénocentrisme, si courant - matériel oblige - se voit ainsi mis entre parenthèse, du moins dans les premiers chapitres, au profit d'une vision globalisante: on ne peut qu'applaudir à cet élargissement bienvenu et stimulant, à cette remise en contexte de la production athénienne, même si une telle ouverture ne laisse pas de poser des problèmes méthodologiques $(c f$. infra $)$. La table des matières reflète parfaitement la méthode appliquée : après un premier chapitre consacré aux premières apparitions, dès le VII s. av. J.-C., de figures dionysiaques, chaque chapitre analyse, sous l'angle dionysiaque, un type de vases dans une période chronologique donnée; nous passons ainsi des petits vases de la $1^{\text {re }}$ moitié du VI $\mathrm{e}$. av. J.-C. (chap. 2) aux dinoi et cratères de cette même période (chap. 3), puis aux amphores du VI $\mathrm{VI}^{\mathrm{e}}$. av. J.-C. (chap. 4) pour terminer par les coupes et autres petits vases de la $2^{\mathrm{e}}$ moitié du siècle (chap. 5). Le chapitre 6, qui était le dernier de l'édition originale de 2001, présente un résumé des méthodes et des résultats en tirant des parallèles entre les données glanées au cours de l'analyse et l'histoire socio-religieuse d'Athènes durant le $\mathrm{VI}^{\mathrm{e}} \mathrm{s}$. Le dernier chapitre de l'édition anglaise est une reprise d'un article de l'A. publié en 2001 faisant le point sur les études dionysiaques modernes, occasion pour C. I.-K. de dénoncer les préjugés contemporains et les blocages mentaux concernant la figure de Dionysos qu'une certaine lecture des textes antiques a pu ancrer dans notre esprit.

La classification rigoureuse des images par tranches chronologiques et types de supports pourrait donner l'impression que le livre n'est qu'un catalogue de représentations archä̈ques. Il n'en est rien. À l'image du dernier chapitre, que l'A. aurait dû faire figurer en tête de cette 
nouvelle édition, tout le livre est un manifeste pour nouvelle approche de Dionysos, une approche libérée des a priori littéraires et fondée sur l'analyse approfondie des seules images dionysiaques. L'ouvrage de C. I.-K. est un livre à thèses, proche de l'essai, dans lequel l'A. prend le risque de formuler des hypothèses explicatives, de donner une cohérence de sens à des images de prime abord éparses et divergentes.

S’il est bel et bien toujours question d'hypothèses et non de résultats, au vu de la rareté et de la difficulté d'interprétation du matériel (p. 3), plusieurs idées traversent l'ouvrage, lui donnant sa cohérence thématique et formant une sorte de fil rouge au travers du découpage chronologique et typologique du matériel : le Dionysos des images archaïques y est présenté comme le médiateur par excellence, figure permettant d'exprimer les problèmes d'intégration de tout ordre que connaît la cité archaïque, notamment au temps de Solon (différents statuts des femmes, les jeunes, les artisans, les 'bâtards', etc.); mais Dionysos est également celui qui peut y proposer des solutions intégratives, par le rituel, notamment le rituel semiprivé du banquet. Ce sont les trois chapitres centraux (3-5) qui servent de base à l'élaboration de cette hypothèse de travail, soit l'analyse des grands vases prestigieux (d'usage collectifs) de la $1^{\text {re }}$ moitié du VIe s., des amphores de tout le siècle et des petits vases de sa seconde moitié. De ce noyau interprétatif, nous relèverons la brillante interprétation cosmogonique des noces de Thétis et Pélée sur le Dinos de Sophilos (p. 69-75), qui permet à l'A. d'insister sur la place non pas secondaire mais centrale, au sens propre comme figuré, de la figure de Dionysos dans l'ordre cosmique que ces noces visent justement à maintenir : son rôle de médiateur s'inscrit dans un dialogue entre un 'intérieur' et un 'extérieur', dans une transition entre nature et espace civilisé ou habité dont il est le garant et le principe 'intégrateur'. Dionysos se manifeste par là non comme une divinité étrangère à la société et à la cité, mais bien comme un pivot indispensable à toute intégration à la civilisation et à la société. Il ne saurait dès lors être ce dieu nouveau venu ou marginal que les études du XXe siècle ont trop souvent contribué à déclasser.

C'est toujours dans ce rôle de médiateur et d'intégrateur que Dionysos apparaît sur les amphores du $\mathrm{VI}^{\mathrm{e}}$ siècle. La prise en compte systématique de ce matériel de prime abord déconcertant, où le dieu figure le plus souvent en compagnie d'hommes, jeunes pour la plupart, et de femmes, a le mérite de forcer à la réflexion: qui sont ces hommes et ces femmes? Quels sont leurs statuts? Que fait Dionysos dans un environnement parfaitement 'humain'? Dans cette rencontre, l'A. voit un processus de transition et d'intégration : transition entre la nature sauvage (le lierre), la nature cultivée (la vigne) et la civilisation (le vin et le savoir-boire) qui sert d'allégorie à l'intégration des jeunes hommes à la sphère parfaitement adulte du banquet. L'analyse, qui se poursuit au chapitre suivant sur les coupes de la fin du siècle, met en avant la métamorphose du jeune éphèbe, - caractérisé par son statut d'éromène - en symposiaste, adulte fini, capable de tenir le rôle d'éraste et de participer pleinement au banquet et à tous les registres de la vie sociale, militaire et politique. Cette métamorphose se traduit en image, selon l'A., par la figure du danseur grotesque, forme inachevée et déformée de l'idéal de l'éphèbe : en représentant des danseurs grotesques les peintres feraient allusion à un rituel d'intégration, pratiqué dans le cadre ou en marge du banquet, rituel qui, par la métamorphose de l'éphèbe en danseur malformé et ridicule symboliserait l'entre-deux inconfortable de la transition entre deux stades d'intégration sociale. Le passage de l'éphèbe au danseur grotesque, puis de celui-ci à son prototype mythique (!), le satyre, révèle l'importance de la sexualité dans ces rites de passage, domaine que la critique n'a pas eu le courage d'aborder de face dans les décennies précédentes et auquel l'A. a à cœur de redonner toute sa dimension, sans tabou.

Du côté des femmes, les choses sont plus complexes. C.I.-K. reconnaît plusieurs types de femmes présentes aux côtés de Dionysos : la nymphe (femme sexuellement intéressante, 
à l'intersection de deux sphères, la séduction et le mariage; catégorie propre à l'A.), la fiancée-matrone, la mère de jumeaux, la jeune fille sapphique, la proie des satyres sauvages ou encore la compagne féminine des satyres calmes et 'apprivoisés'. Les rapports entretenus sur les vases entre ces différentes femmes et Dionysos d'une part et les satyres de l'autre révèlent leurs statuts différenciés. En tant que dieu des métamorphoses, Dionysos est garant et acteur de leur intégration dans le tissu social. Tout comme la vigne et le vin, la femme doit être domestiquée et intégrée à la société. Cette iconographie serait à mettre en parallèle direct avec la situation sociale de la cité (en particulier Athènes) au VI siècle; elle serait le reflet d'une réflexion sur les divers statuts des femmes, entre objets de séduction, candidates au mariage et à la procréation légitime, ou encore femmes avec lesquelles il est permis de jouer et de se laisser aller à une sexualité 'gratuite' sans aucun but procréatif et directement social.

Autre rituel d'intégration dont les images seraient un écho indirect : les processions avec un cavalier à la mule. Au travers des différents chapitres et des tranches chronologiques concernées, C.I.-K. analyse l'évolution de ce schéma iconographique, en insistant fort justement sur la flexibilité de sa composition et sur l'identité floue du cavalier qui n'est pas toujours Héphaistos ramené à l'Olympe par Dionysos, loin de là. Voyant ces cortèges à la mule comme le reflet d'un rituel intégratif, faisant encore une fois le lien entre un dehors et un dedans, entre un monde sauvage et la cité civilisée, l'A. propose d'y lire la transcription imagée (dans les deux sens du terme) de la volonté de Solon d'intégrer les classes inférieures à la société athénienne en pleine mutation. Dionysos jouerait là encore son rôle de 'passeur', tandis que les danseurs ou satyres représenteraient cet entre-deux inconfortable et troublant, matérialisant en image le passage et l'intégration.

On le voit, l'idée maitresse selon laquelle Dionysos est le champion et l'acteur de toute intégration s'impose de bout en bout dans cette étude. Cette interprétation n'est pas nouvelle dans l'œuvre abondante de l'A. et l'ouvrage est à ce titre l'aboutissement d'une longue quête patiemment et méthodiquement élaborée depuis de nombreuses années ( $c f$. notamment les sept travaux parus de "Iconografia dionisiaca» auxquels il faut encore ajouter deux travaux sous presse). Cette hypothèse a le mérite d'offrir un point de vue original qui prend ses distances avec les interprétations trop souvent fondées sur les textes. L'argumentation est particulièrement convaincante dans le cas du Dinos de Sophilos ou du répertoire des amphores archaïques et représente un apport précieux et incontournable à la recherche sur Dionysos. On a toutefois l'impression que la recherche de cohérence qui caractérise la démarche analytique de C. I.-K. impose ce schéma interprétatif dans des cas de figure moins évidents. Si le cortège du cavalier à la mule est bien une façon d'exprimer l'intégration des couches inférieures de la population athénienne, on peine à s'expliquer sa présence sur le cratère François, vase on ne peut plus aristocratique. L'analyse approfondie de ce vase d'exception (p. 75-92) laisse une impression de lecture dirigée des scènes pour rendre à tout prix cohérent l'ensemble des thèmes représentés (noces de Thétis et Pélée, Troilos, cortège du cavalier à la mule). De même, si ce type de cortège est bel et bien une création athénienne, reflet d'une volonté de la cité archaïque d'intégrer ses classes défavorisées, on s'interroge sur la présence de ce même type de cortège dans la céramique extraathénienne; l'A. stipule bien que le problème de l'intégration des classes inférieures est une réalité à laquelle sont confrontées toutes les cités concernées. Mais la question n'est pas posée, je crois, dans les termes appropriés : en faisant l'impasse sur l'analyse différenciée du schème et du thème, C.I.-K. brûle les étapes interprétatives. Le schème du cortège avec cavalier à la mule est multiple comme le souligne à juste titre l'A. Il est de ce fait imprudent de ramener ces expressions contrastées à un seul et même thème, imposant par là-même une cohérence factice sur une réalité mouvante. Il conviendrait en outre de s'interroger sur le sens à donner aux reprises de ce motif dans des céramiques autres qu'athéniennes : est-ce le 
schème seul qui se transmet? Ces cortèges ont-ils la même signification, le même référent partout?

C'est à cette même question de différenciation du schème et du thème que l'on se trouve à nouveau confronté dans la lecture des deux premiers chapitres. La recherche des premières manifestations dionysiaques dans l'imagerie mène l'A. à un parcours très large. Le bénéfice d'une telle démarche est indéniable : la figure de Dionysos semble apparaitre dès le VII e siècle sur un 'cratère mélien', alors que des types de danseurs grotesques (ou pansus), bien présents dans la production orientalisante de Corinthe (aryballes), se retrouvent sur une amphore proto-attique du VII et même sur un vase géométrique de Milet. Toutefois la question méthodologique paraît ici sous-estimée par l'A. : ces danseurs que l'on verra par la suite graviter dans l'orbite de Dionysos peuvent-ils déjà être interprétés comme bachiques par leur simple représentation sur des vases à vin ? En d'autres termes, ne devrait-on pas parler simplement d'un schème, répandu dans le bassin égéen (Cyclades, Corinthe, Béotie, Laconie, Attique), avec des variations qu'il vaudrait la peine d'étudier de plus près ? Cette distinction schème-thème éclairerait peut-être la délicate question des relations entre ces danseurs et les satyres, et minimiserait le danger d'une lecture rétroprojective, immanquablement présent dans une étude qui ne peut s'appuyer sur aucun document de type textuel. On regrettera enfin l'absence de prise en compte de la dimension humoristique et ironique des satyres. En prenant très au sérieux les images dionysiaques, l'A. semble se refuser cette 'facilité' : le rire et la dérision ne sont-ils pas eux aussi une composante essentielle du rituel ?

Ces quelques remarques personnelles ne sont qu'une entrée en discussion, non une critique. Le livre de C.I.-K., dont la lecture est très stimulante et vivifiante, est en lui-même une invitation à la réflexion et à la discussion; il n'est malheureusement pas possible de rendre compte ici des nombreuses finesses d'analyse, de la richesse et de la densité du propos. Par son approche résolument personnelle, l’A., à son habitude, nous pousse à sortir de nos schémas interprétatifs, à regarder d'un œil neuf des images que l'on croyait connaitre. Même si certains ne seront peut-être pas entièrement convaincus par toutes les lectures proposées, cet ouvrage marque un jalon capital dans les recherches sur Dionysos. Il est, dans une large mesure, exemplaire de ce qu'une pratique rigoureuse de la lecture d'images, de ce qu'un œil et un esprit bien affûtés par des années de pratique peuvent apporter à la recherche. Le Dionysos des images n'est pas le même que celui dont nous parlent les textes. À créneau d'expression différent, message différent: preuve encore une fois que ce n'est que de la combinaison des différents types de documents qui nous sont parvenus que nous pouvons espérer une compréhension du monde antique.

Anne-Françoise Jaccottet (Université de Neuchâtel)

\section{Philippe MOnBrun, Les Voix d'Apollon. L'arc, la lyre et les oracles, Rennes, Presses Universitaires de Rennes, 2007. 1 vol. 15, $5 \times 24 \mathrm{~cm}, 346$ p. (Coll. Histoire). ISBN : 978-2-7535-0415-8.}

Depuis bientôt dix ans, on s'était fait à l'image insolite d'un Apollon «le couteau à la main ", sorti du laboratoire expérimental de M. Detienne comme un deus ex machina (c.r. dans Kernos 13, 2000, p. 274-277). Or voici que refait surface un Apollon citharède, doté aussi, il est vrai, d'un arc redoutable et proclamant de surcroît sa puissance prophétique, ainsi que le fait voir le vieil hymne homérique qui conte sa nativité. La notion de voix n'est pas sans rappeler un "Apollon sonore » beaucoup plus ancien, celui de G. Dumézil, mais, on va le voir, c'est sur de tout autres bases que la présente enquête s'édifie, prenant cette fois pour objet toute la trilogie sonore qu'énonce le titre, sans s'interdire, chemin faisant, de faire droit à quelques auxiliaires efficaces relevant tantôt du règne végétal, comme le palmier, tantôt du 